ISSN 1980-5098@®@ DOI: http://dx.doi.org/10.5902/1980509831573

\title{
PRODUÇÃO E APORTE DE CARBONO, NITROGÊNIO E FÓSFORO NA SERAPILHEIRA FOLIAR DO PARQUE NACIONAL SERRA DE ITABAIANA
}

\author{
PRODUCTION AND SUPPLY OF CARBON, NITROGEN AND PHOSPHORUS IN THE LEAF \\ LITTER OF NATIONAL PARK SERRA DE ITABAIANA
}

\begin{abstract}
Audenis Fagner de Jesus Nascimento ${ }^{1}$ Tácio de Oliveira da Silva ${ }^{2}$ Renisson Neponuceno Araújo Filho ${ }^{3}$ Everardo Valadares de Sá Barretto Sampaio ${ }^{4}$ Alceu Pedrotti ${ }^{2}$ Maria Isidória Silva Gonzaga ${ }^{2}$ Victor Casimiro Piscoya ${ }^{5}$
\end{abstract}

\section{RESUMO}

A serapilheira é uma das principais vias de retorno de nutrientes no sistema planta-solo-planta e sua determinação permite analisar a reestruturação de ambientes florestais antropizados. O conhecimento da produção da serapilheira é fundamental para determinar o potencial de regeneração de uma área por meio do aporte e ciclagem dos nutrientes. Objetivou-se neste estudo quantificar a produção de serapilheira e o aporte de carbono e dos nutrientes da fração foliar em áreas de ocorrência de Neossolos Quartzarênicos, com vegetação em diferentes estágios de regeneração, no Parque Nacional Serra de Itabaiana, em Sergipe. Em cada uma das áreas de Areias Brancas Abertas (ABA), Areias Brancas Intermediárias (ABI) e Areias Brancas Fechadas (ABF) instalaram-se coletores nos quais a serapilheira foi coletada mensalmente, de janeiro a dezembro de 2011. Em subamostras da serapilheira foram determinadas as quantidades de carbono (C), nitrogênio $(\mathrm{N})$ e fósforo $(\mathrm{P})$. Com a ação do fogo nas áreas de Areias Brancas do Parque da Serra de Itabaiana, ficou evidente que o mesmo altera o estado de equilíbrio dinâmico alcançado pelo ecossistema, logo, as condições nutricionais do solo são modificadas. Os acúmulos de $\mathrm{C}, \mathrm{N}$ e $\mathrm{P}$ não apresentaram variação sazonal significativa, de acordo com a produção da serapilheira em cada área estudada, sendo que a área ABF proporcionou os maiores acúmulos destes elementos ao longo do período de avaliação.

Palavras-chave: Mata Atlântica; perturbação antrópica; matéria orgânica; processo de regeneração.

\begin{abstract}
Litter is a major route of return of nutrients in the system plant-soil -plant and its determination allows to analyze the restructuring of anthropogenic forest environments. Knowledge of litter production is a critical in determining the potential for regeneration of an area through the intake and nutrient cycling. This study aimed to quantify the litter production and the carbon contribution and leaf fraction nutrients in areas of occurrence of Quartzipsamments with vegetation at different stages of regeneration in National Park Serra de Itabaiana in Sergipe, state. In each of the areas of Areia Branca Aberta (ABA), Areia Branca Intermediaria $(\mathrm{ABI})$ and Areia Branca Fechada $(\mathrm{ABF})$, settled collectors where the litter was collected monthly from January to December 2011. In the litter subsamples amounts of carbon (C), nitrogen (N) and

1 Engenheiro Florestal, Mestre em Ciências Florestais, Departamento de Engenharia Florestal, Universidade Federal dos Vales do Jequitinhonha e Mucuri, Rua da Glória, 187, CEP 39100-000, Diamantina (MG), Brasil. audenis_fagner@yahoo.com.br

2 Engenheiro Agrônomo, Dr., Professor do Departamento de Engenharia Agronômica, Universidade Federal de Sergipe, Cidade Universitária, Av. Mal. Rondon s/n, Jardim Rosa Elze, CEP 49100-000, São Cristóvão (SE). alceupedrotti@gmail.com / mariaisisilva@gmail.com

3 Engenheiro Florestal, Dr., Departamento de Engenharia Agronômica, Universidade Federal de Pernambuco, Rua Dom Manoel de Medeiros, s/n, Dois Irmãos, CEP 52171-900, Recife (PE). nepoaraujo@gmail.com

4 Engenheiro Agrônomo, PhD., Professor do Departamento de Energia Nuclear, Universidade Federal de Pernambuco, Av. Prof. Moraes Rego, 1235, Cidade Universitária, CEP 50670-901, Recife (PE).esampaio@ufpe.br

5 Engenheiro Florestal, Dr., Professor do Departamento de Tecnologia Rural, Universidade Federal de Rural de Pernambuco, Rua Dom Manoel de Medeiros, s/n, Dois Irmãos, CEP52171-900,Recife(PE).victorcasimiropiscoya@ gmail.com
\end{abstract}

Recebido para publicação em 21/03/2013 e aceito em 16/08/2016

Ci. Fl., v. 28, n. 1, jan.- mar., 2018 
phosphorus $(\mathrm{P})$ were determined. Along with the action of fire in the areas of Areias Brancas of Serra de Itabaiana Park, it became clear that it changes the state of dynamic equilibrium achieved by the ecosystem, so the nutritional soil conditions are modified. The accumulation of $\mathrm{C}, \mathrm{N}$ and $\mathrm{P}$ showed no significant seasonal variation according to the production of litter in each area studied, and the ABF area provided the largest accumulations of these elements throughout the evaluation period.

Keywords: Atlantic Forest; anthropogenic disturbance; organic matter; regeneration method.

\section{INTRODUÇÃO}

A serapilheira constitui-se na principal via de aporte de carbono, nitrogênio, fósforo e cálcio no sistema solo-planta, representando um componente fundamental na manutenção e no desenvolvimento de um ecossistema florestal, uma vez que influencia a produtividade e o potencial de regeneração da vegetação local (FIGUEIREDO FILHO et al., 2003; SHUMACHER, 2004; COSTA et al., 2010).

Em ambientes florestais, a ciclagem de nutrientes tem sido estudada com o intuito de se obter maior conhecimento da dinâmica dos nutrientes, não só para o entendimento do funcionamento dos ecossistemas, mas também para buscar informações sobre práticas de manejo florestal para a manutenção da biodiversidade em determinado sítio florestal (SOUZA; DAVIDE, 2001).

No Brasil, em determinadas regiões, são encontrados estudos que fornecem informações a respeito da contribuição da serapilheira na ciclagem de nutrientes, a exemplo da região Sudeste (CIANCIARUSO et al., 2006; TERROR; SOUSA; KOZOVITS, 2011), região Norte (FERNADES; NASCIMENTO; CARVALHO, 2007) e, apesar de escassos, da região Nordeste (SANTANA; SOUTO, 2011). Contudo, é necessário incrementar os trabalhos para a avaliação da produção da serapilheira e da ciclagem de nutrientes no ecossistema florestal, para que seja possível avaliar as condições da composição florística e do microclima, além de entender a relação desse compartimento com o processo regenerativo da vegetação (MARTINS; RODRIGUES, 1999), principalmente, nos diversos estádios sucessionais dos remanescentes florestais oriundos dos processos de fragmentação.

O Parque Nacional da Serra de Itabaiana, no estado de Sergipe, possui vegetação exuberante e constantemente é alvo de vários distúrbios antrópicos, como o fogo oriundo das atividades de caça e agropecuária desenvolvidas pela comunidade do entorno. O solo dessa região caracteriza-se por sua baixa fertilidade natural, desta forma, as espécies vegetais necessitam do aporte e manutenção da serapilheira, a qual, através da decomposição da matéria orgânica, contribui para a ciclagem e fornecimento de nutrientes. Estudos a respeito da produção e decomposição da serapilheira nesta área são recentes e não correspondem à necessidade do local.

As formações vegetais florestais do Parque Nacional foram subdivididas em três grandes tipos naturais: Matas Primárias e Matas Secundárias, Areias Brancas e Campos Graminosos, segundo a classificação da vegetação brasileira (IBGE, 1992). Existem, ainda, muitas áreas degradadas com vegetação descaracterizadas das originais (DANTAS; RIBEIRO, 2010). No habitat das Areias Brancas, uma comunidade arbustivo-herbácea escleromorfa é distinta das vegetações de matas estacionais e de Caatinga hipoxerófila, características das regiões do agreste do estado de Sergipe. Nesse sentido, são necessários estudos para se ter conhecimento do potencial da vegetação em se restaurar, após sofrer com a ação antrópica, e as determinações da produção e ciclagem de nutriente da serapilheira são um bom indicador do progresso da recuperação.

As Areias Brancas distribuem-se fragmentadamente, ocupando uma área total estimada de 347 ha, posicionando-se na face leste das serras de Itabaiana e Comprida, entre o domo e as matas de pé de serra (DANTAS; RIBEIRO, 2010). Dantas et al. (2010) reconheceram fisionomias distintas de Areias Brancas na Serra de Itabaiana, as quais variam em estádios sucessionais, dependendo do histórico de degradação da área.

Adegradação afeta negativamente as condições de preservação, com o consequente comprometimento da qualidade ambiental, sendo necessário o desenvolvimento de modelos para a recuperação da vegetação que considerem a reabilitação de suas características estruturais e funcionais, relacionadas principalmente à estabilidade do solo, retenção de nutrientes, alta produtividade, habitat para ocupação e regeneração de espécies e a regulação da qualidade do ecossistema (RODRIGUES; GANDOLFI, 2000). 
Diante do exposto, o objetivo neste estudo foi quantificar a produção de serapilheira e o aporte de carbono e dos nutrientes na fração foliar em áreas de ocorrência de Neossolos Quartzarênicos presentes em ecossistemas frágeis, com vegetação em diferentes estágios de regeneração, no Parque Nacional Serra de Itabaiana, em Sergipe.

\section{MATERIAL E MÉTODOS}

\section{Localização e descrição da área}

O estudo foi realizado no Parque Nacional Serra de Itabaiana, em Sergipe, localizado no limite entre os municípios de Itabaiana, Areia Branca e Campo do Brito, em torno da latitude $10^{\circ} 40^{\prime} \mathrm{S}$, longitude $37^{\circ} 25^{\prime} \mathrm{O}$ e altitude aproximada de $670 \mathrm{~m}$ (VICENTE et al.,2005). Este Parque constitui uma Unidade de Conservação de Proteção Integral, representada por uma região de ecótono entre Mata Atlântica e Caatinga, na qual partes das suas características mantém-se preservada.

O clima característico da região é do tipo As' - clima tropical com verão seco e moderado excedente hídrico no inverno, segundo a classificação de Köppen. De acordo com Dantas et al. (2010), a precipitação pluvial média anual concentra-se na faixa entre 1.100 a $1.300 \mathrm{~mm}$ e evapotranspiração anual média é de $800 \mathrm{~mm}$. O período chuvoso ocorre entre março a agosto, e o período seco apresenta-se com deficit hídrico ocorrendo entre os meses de setembro e fevereiro. A precipitação mensal do período avaliado foi obtida na base de dados do Programa de Monitoramento Climático em Tempo Real da Região Nordeste (PROGRAMA DE MONITORAMENTO CLIMÁTICO EM TEMPO REAL DA REGIÃO NORDESTE, 2012).

Na região avaliada, o tipo de solo predominante é o Neossolo Quartzarênico formado por areias quartzosas distróficas, com elevada drenagem, moderado a extremamente ácido e de baixa fertilidade natural (EMBRAPA, 1975; 2006). Esses solos apresentam textura arenosa e baixa capacidade de agregação de partículas, condicionados pelos baixos teores de argila e de matéria orgânica.

Para a realização do estudo foram selecionadas áreas localmente denominadas de Areias Brancas, formadas por uma vegetação escleromorfa com componentes que variam de arbustivo-herbácea a arbustivo-arbórea, diferente das vegetações de matas estacionais e da caatinga hipoxerófila, características das regiões de agreste de Sergipe, e similares fisionomicamente e floristicamente às restingas do estado (DANTAS et al., 2010).

Três áreas com histórico de perturbação pelo fogo semelhante, porém, com diferentes tempos de regeneração pós-distúrbio foram escolhidas (WHITE; RIBEIRO, 2011). Areias Brancas Abertas (ABA)localizada na Serra Cumprida que sofreu perturbação mais recente, atingida por um grande incêndio provocado em 2005, representando a área em estádio inicial de sucessão. O solo deste habitat é recoberto por alta densidade de gramíneas e vegetação lenhosa formada por arbustos distribuídos isoladamente ou em pequenos grupos, sob os quais se concentra a serapilheira. Areia Branca Intermediária (ABI) - localizada na Serra de Itabaiana e protegida desde 1978, porém com ocorrência de incêndios periódicos até 1994, representando a área em estádio de sucessão intermediária. A fisionomia da vegetação é composta por três estratos: arvoretas isoladas, de aproximadamente 2,0 metros de altura, arbustos esparsos de 1,0-1,5 metro de altura e pequenos arbustos de 0,5-1,0 metro de altura. A superfície do solo é recoberta em menor densidade por gramíneas e a vegetação lenhosa apresenta-se mais desenvolvida que na área anterior. Areia Branca Fechada (ABF) - localizada na Serra de Itabaiana, sem vestígios e notícias de incêndios há mais de 30 anos, sendo considerada a área mais preservada e em estádio de sucessão mais avançada. Esta área é formada continuamente por vegetação arbórea e arbustiva, em que a mata é mais extensa e estruturada que as duas outras áreas. (DANTAS; RIBEIRO, 2010).

A Tabela 1 apresenta a análise química dos solos das três áreas de Areias Brancas avaliadas no Parque Nacional da Serra de Itabaiana, Sergipe. 
TABELA 1: Análise química dos solos das áreas de Areias Brancas Abertas (ABA), Areias Brancas Intermediárias (ABI) e Areias Brancas Fechadas (ABF) do Parque Nacional Serra de Itabaiana, Sergipe, Brasil.

TABLE 1: Chemical analysis of soil in the areas of Areia Branca Aberta (ABA), Areia Branca Intermediaria (ABI) and Areia Branca Fechada (ABF) of Serra de Itabaiana National Park, Sergipe state, Brazil.

\begin{tabular}{cccccccccccccc}
\hline Área & $\mathrm{pH}\left(\mathrm{H}_{2} \mathrm{O}\right)$ & \multicolumn{1}{c}{$\mathrm{COT}$} & $\mathrm{NT}$ & $\mathrm{C} / \mathrm{N}$ & $\mathrm{P}$ & $\mathrm{Ca}^{2+}$ & $\mathrm{K}^{+}$ & $\mathrm{Mg}^{2+}$ & $\mathrm{Al}^{1+}$ & $\mathrm{H}+\mathrm{Al}$ & $\mathrm{CTC}$ & $\mathrm{SB}$ & $\mathrm{V}$ \\
\hline- & - & \multicolumn{2}{c}{$\mathrm{g} \mathrm{kg}^{-1}$} & \multicolumn{2}{c}{$\mathrm{Mgdm}^{-3}$} \\
\hline ABA & 5,17 & 12,84 & 0,35 & 36,09 & 24,47 & 2,58 & 0,0038 & 0,56 & 0,28 & 3,72 & 3,44 & 3,15 & 48,56 \\
$\mathrm{ABI}$ & 5,42 & 2,92 & 0,14 & 19,20 & 24,69 & 0,38 & 0,0020 & 0,00 & 0,13 & 1,26 & 0,51 & 0,37 & 29,36 \\
$\mathrm{ABF}$ & 5,37 & 7,72 & 0,32 & 23,18 & 36,27 & 1,15 & 0,0035 & 0,64 & 0,22 & 2,52 & 2,02 & 1,80 & 47,59 \\
\hline
\end{tabular}

Em que: Carbono Orgânico Total $=(\mathrm{COT})$; Nitrogênio Total $=(\mathrm{NT})$; Relação Carbono/Nitrogênio $=(\mathrm{C} / \mathrm{N})$; Capacidade de Troca Catiônica $=(\mathrm{CTC})$; Soma de Bases $=(\mathrm{SB})$; Saturação de Bases $=(\mathrm{V})$.

\section{Produção de serapilheira e acúmulo de nutrientes}

As coletas da serapilheira foram realizadas mensalmente, de janeiro a dezembro de 2011, em um total de 15 coletores por área. Estes coletores com formato quadrangular e área de $0,25 \mathrm{~m}^{2}$ foram produzidos com tela de náilon (malha de $1 \mathrm{~mm}$ de abertura), sendo fixados com hastes de madeira a uma altura de 0,5 $\mathrm{m}$ do solo. Em cada área, os coletores foram colocados a cada $12,5 \mathrm{~m}$ ao longo de dois transectos paralelos de $100 \mathrm{~m}$, distando $20 \mathrm{~m}$ entre si.

O material foliar interceptado nos coletores foi acondicionado em embalagens de papel, e conduzido ao laboratório para análise. Posteriormente, o material foi seco em estufa e pesado em balança de precisão para obter a massa seca do componente vegetal.

Mensalmente, retirou-se de cada coletor uma amostra representativa do material vegetal visando determinar as quantidades de carbono (C) pelo método de oxidação por dicromato juntamente com uma fonte externa de aquecimento; nitrogênio $(\mathrm{N})$ determinado pelo método Microkjeldahl; e fósforo (P) determinado por colorimetria, de acordo com a metodologia descrita por Malavolta, Vitti e Oliveira (1997).

$\mathrm{O}$ acúmulo dos elementos na serapilheira foliar foi obtido pelo produto da biomassa foliar pelos teores dos elementos. As variáveis analisadas foram: produtividade de serapilheira foliar, acúmulo de carbono e dos nutrientes, nitrogênio e fósforo.

Os dados foram submetidos à análise de variância e as médias, quando significativas, foram comparadas pelo teste Tukey, a 5\% de probabilidade. Foi realizada a análise de correlação linear de Pearson entre a serapilheira foliar produzida e a precipitação pluvial, usando o programa Statistica 8.0 (STATSOFT, 2008). Para a comparação temporal, os dados foram submetidos à análise de regressão, com a escolha do modelo quadrático que melhor se ajustou pelo teste $\mathrm{F}$, a $5 \%$ de probabilidade, e pelo coeficiente de determinação $\left(\mathrm{R}^{2}\right)$, de acordo com Banzatto e Kronka (2006). As análises estatísticas foram realizadas com o uso do programa estatístico SISVAR (FERREIRA, 2011).

\section{RESULTADOS E DISCUSSÃO}

\section{Produção de serapilheira foliar}

$\mathrm{Na}$ comparação entre as três áreas de estudo, verificou-se que a área $\mathrm{ABF}$ concentrou a maior produção de serapilheira foliar, $2.679 \mathrm{~kg} \mathrm{ha}^{-1}$, enquanto as áreas ABA e ABI apresentaram produções inferiores, com $1.256 \mathrm{~kg} \mathrm{ha}^{-1} \mathrm{e} 1.112 \mathrm{~kg} \mathrm{ha}^{-1}$, respectivamente. Diante disto, os resultados apontaram efeito significativo da produção de biomassa foliar de $\mathrm{ABF}$ em relação às áreas $\mathrm{ABA}$ e $\mathrm{ABI}$, principalmente nos meses de menores precipitações pluviométricas, janeiro e dezembro (Figura 1). Pode-se relacionar tal fato com os diferentes estádios sucessionais da vegetação nas áreas estudadas, já que a ocorrência de alterações imprevisíveis na composição de espécies, ou mesmo nas condições ambientais podem modificar a sequência do processo de sucessão, limitando sua previsibilidade (KREYLING et al., 2011). 


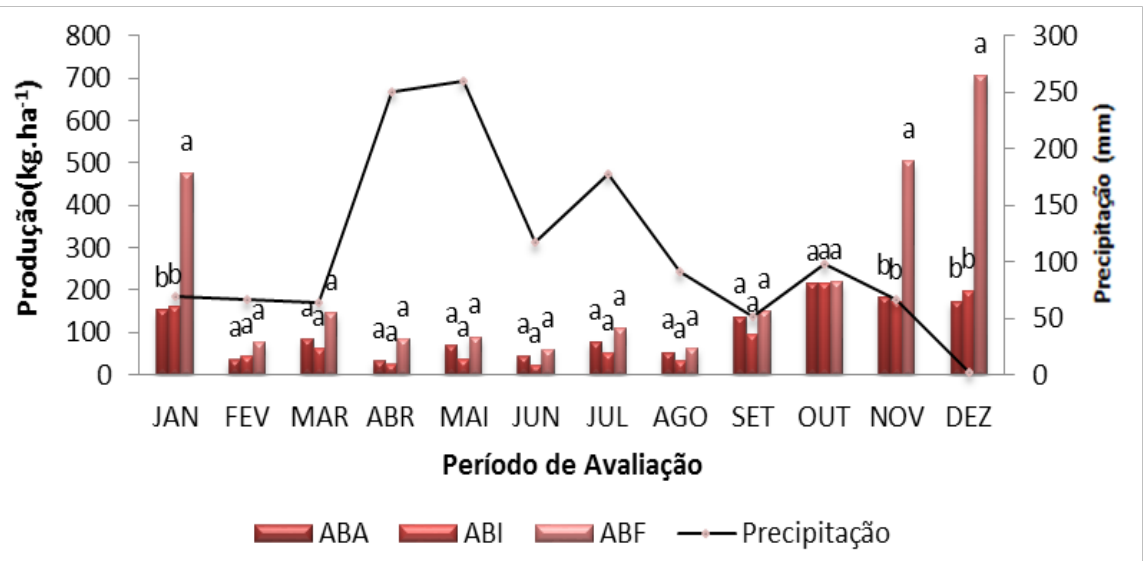

FIGURA 1: Produção de serapilheira foliar nas áreas de Areia Branca Aberta (ABA), Areia Branca Intermediária (ABI) e Areia Branca Fechada (ABF) no Parque Nacional Serra de Itabaiana ao longo de 2011. Médias iguais dentro de cada mês não diferem entre si a $5 \%$ de probabilidade.

FIGURE 1: Production of leaf litter in the areas of Areia Branca Aberta (ABA), Areia Branca Intermediaria (ABI) and Areia Branca Fechada (ABF) in National Park of Serra Itabaiana over the period of 2011. Equal means with in each month not differ at $5 \%$ probability.

Ao relacionar os valores de produção encontrados mensalmente com os dados de precipitação, ficou evidenciado que o período de novembro a janeiro representou uma época de maior produção, sendo esse período correspondente à estação seca, na qual a área $\mathrm{ABF}$ destacou-se com os maiores valores de produção, quando comparada com as demais áreas (Figura 1). Cabe informar que as maiores perdas de folhas nesta época também ocorreram em virtude do sistema de defesa das plantas que na sua maioria são caducifólia e semicaducifólia que, quando passam por estresse hídrico, tendem a perder suas folhas parcial ou totalmente.

A maior deposição da biomassa foliar no final da estação seca pode ser uma resposta da vegetação ao estresse hídrico ocorrido, uma vez que a queda das folhas reduziria a perda de água por transpiração (MARTINS; RODRIGUES, 1999). Os valores elevados da fração folha evidenciam uma sazonalidade mais pronunciada (MORELLATO, 1993).

Entretanto, ao correlacionar os valores de deposição mensal de serapilheira com as taxas de precipitação, observou-se que no geral não houve correlação (Pearson, $\mathrm{p}<0,05$ ), indicando, assim, que a sazonalidade não foi o principal fator na produção de serapilheira, fato este que pode estar relacionado ao curto intervalo de tempo de coleta, que pode ter influenciado para variações atípicas no período coletado (Tabela 2).

TABELA 2: Correlação de Pearson $(p<0,05)$ entre a serapilheira foliar produzida e a precipitação pluvial para as áreas de Areias Brancas, no Parque Nacional Serra de Itabaiana, Itabaiana - SE.

TABLE 2: Pearson correlation $(\mathrm{p}<0.05)$ between the produced leaf litter and rainfall in the areas of Areias Brancas in the National Park Serra de Itabaiana, Itabaiana, SE state.

\begin{tabular}{cccc}
\hline \multirow{2}{*}{ Variável Climática } & \multicolumn{3}{c}{ Área } \\
\cline { 2 - 4 } & ABA & ABI & ABF \\
\hline Precipitação & $-0,508674^{\mathrm{NS}}$ & $-0,558489^{\mathrm{NS}}$ & $-0,561075^{\mathrm{NS}}$
\end{tabular}

Em que: ${ }^{\mathrm{NS}}=$ Não apresenta significância a 5\% de probabilidade.

Ao relacionar o período avaliado com a dinâmica de produção da serapilheira foliar nas três áreas de Areias Brancas por meio de equação polinomial quadrático, foi verificada a existência de relação significativa entre tempo X produção (Tabela 3). Isso acontece em função do modelo de sucessão da vegetação pós-distúrbio que ocorre, caracterizado pela substituição de espécies, já que as espécies pioneiras escleromorfas ao longo do tempo são substituídas gradualmente por espécies menos escleromorfas, em um processo lento de convergência da composição florística em direção à estruturação anterior ao distúrbio, influenciado pelo aumento do teor nutricional dos solos ocasionados pelo gradual acúmulo de matéria orgâ- 
nica nos mesmos (KEELEY; FOTHERINGHAM; BAER-KEELEY, 2005).

Deste modo, os resultados demonstraram que a área $\mathrm{ABF}$ proporcionou uma maior produção de serapilheira, quando comparada com as outras áreas, em virtude do seu estádio sucessional mais avançado associado às condições ambientais e condições edáficas favoráveis.

TABELA 3: Produção Total de serapilheira nas áreas de Areia Branca Aberta (ABA), Areia Branca Intermediaria (ABI) e Areia Branca Fechada (ABF) no Parque Nacional Serra de Itabaiana ao longo de 12 meses de avaliação.

TABLE 3: Total production of litter in the areas of Areia Branca Aberta (ABA), Areia Branca Intermediaria (ABI) and Areia Branca Fechada (ABF) in Parque Nacional Serra de Itabaiana over 12 months evaluation.

\begin{tabular}{ccc}
\hline Área & Equação & $\mathrm{R}^{2}(\%)$ \\
\hline ABA & $\mathrm{y}=3,2486 \mathrm{x}^{2}-31,801 \mathrm{x}+135,44$ & $65,24^{*}$ \\
ABI & $\mathrm{y}=4,7199 \mathrm{x}^{2}-50,785 \mathrm{x}+167,12$ & $76,92^{*}$ \\
ABF & $\mathrm{y}=16,353 \mathrm{x}^{2}-187,48 \mathrm{x}+556,14$ & $87,68^{*}$ \\
\hline
\end{tabular}

Em que: Significância a 5\% de probabilidade.

Os dados observados mostram-se mais relevantes quando comparados os acúmulos de serapilheira com os diferentes estádios de sucessão pós-distúrbio. As áreas ABA e ABI sofreram perturbações mais recentes, logo apresentam estádios de sucessão inicial e intermediário, respectivamente, o que refletiu nos baixos valores mensais de serapilheira. Além disto, essas áreas são formadas por uma vegetação que varia de arbustivo-herbáceo com árvores de pequeno porte e menos densas. Já a área $\mathrm{ABF}$, que compreende uma fisionomia florestal formada por um dossel mais desenvolvido, contribuiu para uma maior produção de serapilheira. Adicionado a isso, os maiores teores de nutrientes do solo encontrados nesta área (Tabela 1), podem ser efeito da absorção de camadas mais profundas do solo pelas raízes das plantas e posterior ciclagem de nutrientes para a superfície do solo pela deposição de serapilheira (JOGGABY; JACKSON, 2004).

\section{Estoque de carbono}

Obteve-se um estoque de carbono via serapilheira foliar de $454 \mathrm{~kg} \mathrm{ha}^{-1}$ para ABA; $400 \mathrm{~kg} \mathrm{ha}^{-1}$ para $\mathrm{ABI}$ e $971 \mathrm{~kg} \mathrm{ha}^{-1} \mathrm{em} \mathrm{ABF}$ (Tabela 4). Este acúmulo de carbono nas três áreas relaciona-se com a produção de serapilheira de forma significativa, logo, quanto maior a quantidade de serapilheira, maior será a quantidade de material orgânico proveniente da vegetação. Assim, pode-se afirmar que a quantidade de carbono pode estar relacionada com o porte da vegetação e do seu estado de conservação.

TABELA 4: Estoque de carbono na serapilheira foliar nas áreas de Areia Branca Aberta (ABA), Areia Branca Intermediaria (ABI) e Areia Branca Fechada (ABF), no Parque Nacional Serra de Itabaiana ao longo de 2011.

TABLE 4: Carbon stock in the leaf litters in the areas of Areia Branca Aberta (ABA), Areia Branca Intermediária (ABI) and Areia Branca Fechada (ABF), at National Park of Serra Itabaiana over the period of 2011.

\begin{tabular}{cccc}
\hline \multirow{2}{*}{ Mês } & ABA & ABI & ABF \\
\cline { 2 - 4 } & & $\mathrm{C}\left(\mathrm{kg} \mathrm{ha}^{-1}\right)$ & \\
\hline Janeiro & $60,31 \mathrm{~b}^{*}$ & $62,32 \mathrm{~b}$ & $187,9 \mathrm{a}$ \\
Fevereiro & $14,60 \mathrm{a}$ & $16,65 \mathrm{a}$ & $29,59 \mathrm{a}$ \\
Março & $21,56 \mathrm{a}$ & $54,98 \mathrm{a}$ \\
Abril & $30,15 \mathrm{a}$ & $9,18 \mathrm{a}$ & $34,25 \mathrm{a}$ \\
Maio & $11,04 \mathrm{a}$ & $13,96 \mathrm{a}$ & $37,35 \mathrm{a}$ \\
Junho & $27,45 \mathrm{a}$ & $8,32 \mathrm{a}$ & $21,18 \mathrm{a}$ \\
Julho & $14,46 \mathrm{a}$ & $18,57 \mathrm{a}$ & $41,19 \mathrm{a}$ \\
\hline
\end{tabular}

Continua... 
TABELA 4: Continuação...

TABLE 4: Continued...

\begin{tabular}{cccc}
\hline Mês & ABA & ABI & ABF \\
\cline { 2 - 4 } & & C (kg ha $\left.{ }^{-1}\right)$ & $22,91 \mathrm{a}$ \\
Agosto & $20,03 \mathrm{a}$ & $12,06 \mathrm{a}$ & $56,82 \mathrm{a}$ \\
Setembro & $51,39 \mathrm{a}$ & $34,11 \mathrm{a}$ & $79,64 \mathrm{a}$ \\
Outubro & $71,81 \mathrm{a}$ & $81,86 \mathrm{a}$ & $174,95 \mathrm{a}$ \\
Novembro & $66,43 \mathrm{a}$ & $57,86 \mathrm{a}$ & $230,36 \mathrm{a}$ \\
Dezembro & $57,09 \mathrm{~b}$ & $63,82 \mathrm{~b}$ & \\
\hline
\end{tabular}

Em que: Médias iguais dentro de cada mês não diferem entre si a 5\% de probabilidade.

Nota-se que houve interação significativa entre as áreas de Areias Brancas estudadas e o período de avaliação para o acúmulo de $\mathrm{C}$ na serapilheira foliar (Tabela 5). Para todas as áreas estudadas, a curva do acúmulo de $\mathrm{C}$ apresentou um comportamento polinomial quadrático, sendo que a área $\mathrm{ABF}$ obteve o melhor ajuste da equação, como pode ser visto através do coeficiente de determinação ( $\left.\mathrm{R}^{2}\right)$ (Tabela 5). Este maior acúmulo de carbono observado em $\mathrm{ABF}$ se deve à influência interativa do fator fogo com a estrutura da vegetação associada ao tempo decorrido do processo sucessional.

TABELA 5: Estoque de carbono na serapilheira foliar nas áreas de Areias Brancas, aberta (ABA), intermediária (ABI) e fechada (ABF) no Parque Nacional Serra de Itabaiana no período 12 meses de avaliação.

TABLE 5: Carbon stock in the leaf litter in the areas of White Sands, open (ABA), intermediate (ABI) and closed $(\mathrm{ABF})$ in national park of Serra Itabaiana during 12 months of evaluation.

\begin{tabular}{ccc}
\hline Área & $\begin{array}{c}\text { Equação } \\
\text { Estoque de C }\left(\mathrm{kg} \mathrm{ha}^{-1}\right)\end{array}$ & $\mathrm{R}^{2}(\%)$ \\
\hline ABA & $\mathrm{y}=1,1485 \mathrm{x}^{2}-11,723 \mathrm{x}+51,869$ & $60,02 *$ \\
ABI & $\mathrm{y}=1,6166 \mathrm{x}^{2}-17,733 \mathrm{x}+61,063$ & $67,96^{*}$ \\
ABF & $\mathrm{y}=5,6877 \mathrm{x}^{2}-66,816 \mathrm{x}+207,15$ & $85,34^{*}$ \\
\hline
\end{tabular}

Em que: Significância a 5\% de probabilidade.

À medida que as florestas se tornam mais senis, o acúmulo de carbono total no sistema é mais expressivo quando comparado a florestas em idades jovens, com maior ritmo de crescimento (SEDJO; SOHNGEN; MENDELSOHN, 2001). Considerando os valores totais mensais de serapilheira foliar, percebe-se que os estoques de carbono apresentaram a mesma tendência percentual de distribuição. Dessa forma, principalmente devido à grande produção, a serapilheira parece desempenhar papel fundamental no retorno de carbono nas áreas estudadas, representando importante via de ciclagem interna para este elemento.

\section{Estoque de nitrogênio}

A deposição mensal de nitrogênio pela serapilheira foliar nas áreas de Areia Branca está apresentada na Tabela 6. O conteúdo anual total de nitrogênio foi de $40 \mathrm{~kg} \mathrm{ha}^{-1}$, sendo a Areia Branca Fechada, a área com o maior retorno deste nutriente com $25 \mathrm{~kg} \mathrm{ha}^{-1}$. Os meses de maior retorno do nitrogênio coincidem com os períodos de maior deposição da serapilheira, ocorrendo assim a maior deposição de nutrientes no solo. Esses resultados são semelhantes aos de Montagninie e Jordan (2005) que propuseram um valor médio para o fluxo anual de nutrientes, através da serapilheira, em torno de $42,0 \mathrm{~kg} \mathrm{ha}^{-1}$ para N e 2,6 kg ha-1 para $\mathrm{P}$ em solos de fertilidade extremamente baixa. 
TABELA 6: Acúmulo de Nitrogênio na serapilheira foliar nas áreas de Areia Branca Aberta (ABA), Areia Branca Intermediaria (ABI) e Areia Branca Fechada (ABF), no Parque Nacional Serra de Itabaiana ao longo do período de 2011.

TABLE 6: Accumulation of nitrogen in leaf litter in the areas of Areia Branca Aberta (ABA), Areia Branca Intermediaria (ABI) and Areia Branca Fechada (ABF), National Park of Serra Itabaiana over the period of 2011.

\begin{tabular}{cccc}
\hline \multirow{2}{*}{ Mês } & ABA & ABI & ABF \\
\cline { 2 - 4 } & & $N\left(k^{-1}\right)$ & $3,6327 \mathrm{a}$ \\
Janeiro & $0,9894 \mathrm{~b}^{*}$ & $0,9768 \mathrm{~b}$ & $0,6355 \mathrm{a}$ \\
Fevereiro & $0,2365 \mathrm{a}$ & $0,2442 \mathrm{a}$ & $1,1218 \mathrm{a}$ \\
Março & $0,5837 \mathrm{a}$ & $0,4382 \mathrm{a}$ & $0,7195 \mathrm{a}$ \\
Abril & $0,1987 \mathrm{a}$ & $0,1391 \mathrm{a}$ & $0,7678 \mathrm{a}$ \\
Maio & $0,4995 \mathrm{a}$ & $0,2152 \mathrm{a}$ & $0,5177 \mathrm{a}$ \\
Junho & $0,3861 \mathrm{a}$ & $0,1432 \mathrm{a}$ & $0,9302 \mathrm{a}$ \\
Julho & $0,502 \mathrm{a}$ & $0,2617 \mathrm{a}$ & $0,5584 \mathrm{a}$ \\
Agosto & $0,3317 \mathrm{a}$ & $0,2498 \mathrm{a}$ & $1,7561 \mathrm{a}$ \\
Setembro & $0,8255 \mathrm{a}$ & $0,6654 \mathrm{a}$ & $2,1458 \mathrm{a}$ \\
Outubro & $1,3058 \mathrm{a}$ & $1,2877 \mathrm{a}$ & $5,1491 \mathrm{a}$ \\
Novembro & $0,9428 \mathrm{a}$ & $1,0405 \mathrm{a}$ & $7,5412 \mathrm{a}$ \\
Dezembro & $1,0505 \mathrm{a}$ & $1,2874 \mathrm{a}$ & \\
\hline
\end{tabular}

Em que: Médias iguais dentro de cada mês não diferem entre si a 5\% de probabilidade.

A relação entre $\mathrm{C}$ e $\mathrm{N}$ pode ser utilizada como indicador da qualidade nutricional da serapilheira, sendo que valores maiores que 25 indicam que o material foliar apresenta uma baixa proporção de $\mathrm{N}$, limitando assim o processo de decomposição (LUIZÃO et al., 2004). Os valores observados neste estudo, ABA $(\mathrm{C} / \mathrm{N}=58), \mathrm{ABI}(\mathrm{C} / \mathrm{N}=57)$ e $\mathrm{ABF}(\mathrm{C} / \mathrm{N}=38)$ indicam que a baixa qualidade nutricional da serapilheira pode ser um importante fator limitante da decomposição do material foliar depositado nas Areias Brancas, além de refletir em maior eficiência por parte da vegetação na conservação de $\mathrm{N}$ em seus tecidos vivos em relação a outros ecossistemas florestais tropicais.

Ao associar a relação $\mathrm{C} / \mathrm{N}$ da serapilheira coletada com a relação $\mathrm{C} / \mathrm{N}$ do solo, percebe-se que em ambas, a área $\mathrm{ABA}$ apresentou valores maiores que as demais áreas. Este maior valor de $\mathrm{C} / \mathrm{N}$ na área aberta, que apresenta estádio sucessional mais recente, pode ser devido ao efeito do fogo na redisponibilização dos nutrientes estocados nos tecidos vegetais (KNICKER, 2007). As concentrações destes elementos pós-fogo tendem a diminuir progressivamente por meio da lixiviação, entretanto, a velocidade de perda é diferente, ficando o C por mais tempo no sistema (HOMANN et al., 2010), ocasionando, desta forma, maiores valores na relação $\mathrm{C} / \mathrm{N}$.

Estudando a produção de serapilheira e ciclagem de nutrientes de uma floresta estacional semidecidual na zona ripária em Botucatu-SP, Vital et al. (2004) também verificaram que a transferência de nutrientes ao solo acompanhava a tendência de deposição da serapilheira.

Quando se relaciona o período de coleta da serapilheira com o acúmulo de nitrogênio presente na serapilheira produzida, verificou-se efeito significativo para o acúmulo de $\mathrm{N}$ entre as áreas avaliadas, apenas no mês de janeiro, em que a área $\mathrm{ABF}$ foi superior às demais áreas (Tabela 6). No mês de outubro foi observado o maior acúmulo de $\mathrm{N}$ na serapilheira foliar em $\mathrm{ABA}$ e $\mathrm{ABI}$ e dezembro para $\mathrm{ABF}$. Todas as áreas apresentaram o mesmo comportamento em relação ao período, sendo que $\mathrm{ABF}$ acumulou mais $\mathrm{N}$ que as demais (Tabela 7), devido ao tipo de vegetação encontrado na área. 
TABELA 7: Estoque de nitrogênio na serapilheira foliar nas áreas de Areias Brancas, aberta (ABA), intermediária $(\mathrm{ABI})$ e fechada $(\mathrm{ABF})$ no parque nacional Serra de Itabaiana no período 12 meses de avaliação.

TABLE 7: Stocks of nitrogen in leaf litter in the areas of Areias Brancas, Aberta (ABA), Intermediária (ABI) and Fechada (ABF) in National Park of Serra Itabaiana during 12 months of evaluation.

\begin{tabular}{ccc}
\hline Área & $\begin{array}{c}\text { Equação } \\
\text { Estoque de N }\left(\mathrm{kg} \mathrm{ha}^{-1}\right)\end{array}$ & $\mathrm{R}^{2}(\%)$ \\
\hline ABA & $\mathrm{y}=0,0177 \mathrm{x}^{2}-0,1786 \mathrm{x}+0,855$ & $56,74^{*}$ \\
ABI & $\mathrm{y}=0,0292 \mathrm{x}^{2}-0,3122 \mathrm{x}+1,0253$ & $80,25^{*}$ \\
ABF & $\mathrm{y}=0,1576 \mathrm{x}^{2}-1,7146 \mathrm{x}+4,7289$ & $90,44^{*}$ \\
\hline
\end{tabular}

Em que: Significância 5\% de probabilidade.

A quantidade de $\mathrm{N}$ variou significativamente em função do tempo para as três áreas. Aliado a isso, ajustou-se um modelo quadrático para explicar o decréscimo de $\mathrm{N}$ acumulado ao longo do período de avaliação (Tabela 7). Os resultados obtidos para a variação da quantidade de nitrogênio ao longo do período de avaliação, provavelmente, são devido ao estresse hídrico, que provoca um aumento no fluxo de aminoácidos das raízes para as folhas (CASCARDO; OLIVEIRA; SOARES, 1993).

\section{Estoque de fósforo}

A concentração de $\mathrm{P}$ na serapilheira foliar seguiu a mesma tendência do $\mathrm{N}$, ou seja, nos períodos de maior produção de serapilheira foliar obtiveram-se as maiores quantidades desses nutrientes. As concentrações destes nutrientes nas florestas e nos solos são variáveis, podendo estar relacionados à sua concentração no solo, idade da vegetação, estratificação da floresta e à parte da planta.

$\mathrm{Na}$ Tabela 8, é verificado que nos meses janeiro e fevereiro, nos quais ABF apresentou os maiores acúmulos, houve diferença significativa desta área quando comparada com ABA e ABI, fato este que pode ser explicado pela heterogeneidade deste nutriente, não seguindo um padrão de concentração.

De modo geral, os valores de $\mathrm{P}$ obtidos no período chuvoso foram inferiores aos outros elementos avaliados. Como grande parte da serapilheira foliar é formada por folhas mais velhas, a concentração desse elemento é menor. Isso porque o fósforo, por ser constituinte de compostos complexos, ricos em energia, é facilmente retranslocado dos tecidos mais velhos para os mais novos da planta (SANTANA, 2005).

TABELA 8: Estoque de fósforo na fração folha da serapilheira no parque Nacional Serra de Itabaiana, ao longo do período de avaliação nas áreas de Areias Brancas.

TABLE 8: Stock fraction of phosphorus in leaf litter at the National Park of Serra Itabaiana throughout the evaluation period in the areas of Areias Brancas.

\begin{tabular}{cccc}
\hline Mês & ABA & ABI & ABF \\
\cline { 2 - 4 } & & $\mathrm{P}\left(\mathrm{kg} \mathrm{ha}^{-1}\right)$ & \\
\hline Janeiro & $0,0106 \mathrm{ab}^{*}$ & $0,0093 \mathrm{~b}$ & $0,0267 \mathrm{a}$ \\
Fevereiro & $0,0012 \mathrm{~b}$ & $0,0016 \mathrm{~b}$ & $0,0453 \mathrm{a}$ \\
Março & $0,002 \mathrm{a}$ & $0,0049 \mathrm{a}$ \\
Abril & $0,003 \mathrm{a}$ & $0,0007 \mathrm{a}$ & $0,002 \mathrm{a}$ \\
Maio & $0,0016 \mathrm{a}$ & $0,0006 \mathrm{a}$ & $0,0015 \mathrm{a}$ \\
Junho & $0,0019 \mathrm{a}$ & $0,0002 \mathrm{a}$ & $0,0006 \mathrm{a}$ \\
Julho & $0,0005 \mathrm{a}$ & $0,0005 \mathrm{a}$ & $0,0011 \mathrm{a}$ \\
Agosto & $0,0009 \mathrm{a}$ & $0,0012 \mathrm{a}$ & $0,0013 \mathrm{a}$ \\
Setembro & $0,0011 \mathrm{a}$ & $0,0009 \mathrm{a}$ & $0,0029 \mathrm{a}$ \\
Outubro & $0,0025 \mathrm{a}$ & $0,0021 \mathrm{a}$ & $0,0025 \mathrm{a}$ \\
Novembro & $0,0021 \mathrm{a}$ & $0,0016 \mathrm{a}$ & $0,0069 \mathrm{a}$ \\
Dezembro & $0,0034 \mathrm{a}$ & $0,0024 \mathrm{a}$ & $0,0065 \mathrm{a}$ \\
\hline
\end{tabular}

Em que: Médias iguais dentro de cada mês, avaliando as áreas em regeneração não diferem entre si a 5\% de probabilidade. 
Esses resultados indicam que o P é um elemento limitante, pouco disponível naturalmente nos solos para as plantas (Tabela 8). Na Floresta Nacional Mario Xavier, Seropédica - RJ que constitui uma área com floresta de sucessão secundária espontânea, foi realizado estudo no qual se verificaram os valores de $149 \mathrm{~kg}$ $\mathrm{ha}^{-1} \mathrm{ano}^{-1}$ de nitrogênio e $3 \mathrm{~kg} \mathrm{ha}^{-1}$ ano de fósforo (FERNANDES; NASCIMENTO; CARVALHO, 2007), o que confirma a baixa disponibilidade deste elemento em ecossistemas florestais.

Quanto à interação entre a área estudada e o período de avaliação, para o acúmulo de serapilheira, foram utilizadas equações polinomiais, nas quais se ajustou uma regressão com modelo quadrático para cada área. Todas as áreas apresentaram baixa significância, podendo ser notado pelo baixo valor do coeficiente de determinação P (Tabela 9).

TABELA 9: Estoque de fósforo na serapilheira foliar nas áreas de Areias Brancas, aberta (ABA), intermediária $(\mathrm{ABI})$ e fechada $(\mathrm{ABF})$ no parque nacional Serra de Itabaiana no período de 12 meses de avaliação.

TABLE 9: Stock of phosphorus in leaf litter in the areas of White Sands, open (ABA), intermediate (ABI) and closed (ABF) in National Park of Serra Itabaiana during 12 months of evaluation.

\begin{tabular}{ccc}
\hline Área & $\begin{array}{c}\text { Equação } \\
\text { Estoque de } P\left(\mathrm{~kg} \mathrm{ha}^{-1}\right)\end{array}$ & $\mathrm{R}^{2}(\%)$ \\
\hline ABA & $\mathrm{y}=0,0001 \mathrm{x}^{2}-0,0022 \mathrm{x}+0,009$ & $52,24^{*}$ \\
ABI & $\mathrm{y}=0,0002 \mathrm{x}^{2}-0,0023 \mathrm{x}+0,0083$ & $64,13^{*}$ \\
ABF & $\mathrm{y}=0,0007 \mathrm{x}^{2}-0,0114 \mathrm{x}+0,0436$ & $63,37^{*}$ \\
\hline
\end{tabular}

Em que: Significância a 5\% de probabilidade.

Nota-se, assim, uma baixa relação entre os teores de fósforo e o período de avaliação nas três áreas de estudo. Isso pode ser explicado pela baixa quantidade de nutrientes nas folhas, pelo estádio sucessional da floresta e, principalmente, devido aos fatores climáticos, os quais resultam em uma decomposição lenta ou acelerada, dependendo das características ambientais do local.

\section{CONCLUSÕES}

O estudo apresentado neste artigo confirma a importância de pesquisas relacionadas aos ecossistemas florestais e sua capacidade de regeneração em face dos efeitos de fatores antrópicos, como o fogo, no processo de sucessão ecológica.

Com a ação do fogo nas áreas de Areias Brancas do Parque da Serra de Itabaiana, ficou evidente que o mesmo altera o estado de equilíbrio dinâmico alcançado pelo ecossistema, logo, as condições nutricionais do solo são modificadas. Além disso, as queimadas consomem a serapilheira depositada sobre o chão da floresta e, consequentemente, promovem a eliminação da biomassa acima do solo, dessa forma, a baixa qualidade nutricional da serapilheira foliar, influenciada pela baixa fertilidade dos solos avaliados, deve limitar o processo de ciclagem de nutrientes, diminuindo consideravelmente a velocidade com que os nutrientes da serapilheira foliar retornam ao solo e se tornam novamente disponíveis para a vegetação.

Os acúmulos de C, N e P não apresentaram variação sazonal significativa de acordo com a produção da serapilheira em cada área estudada, sendo que a área $\mathrm{ABF}$ proporcionou os maiores acúmulos destes elementos ao longo do período de avaliação.

\section{AGRADECIMENTOS}

Ao amigo, professor e pesquisador Dr. Tácio Oliveira da Silva (in memoriam) por sua inestimável ajuda e contribuição para esta pesquisa.

\section{REFERÊNCIAS}

BANZATTO, D. A.; KRONKA, S. N. Experimentação agrícola. 4.ed. Jaboticabal: FUNEP, 2006. 237p. CASCARDO, J. C. M.; OLIVEIRA, L. E. M.; SOARES, A. M. Disponibilidade de água e doses de gesso 
agrícola nas relações hídricas da seringueira. Revista Brasileira de Fisiologia Vegetal, São Carlos, v. 5, n. 1, p. 31-34, jun. 1993.

CIANCIARUSO, M. V. et al. Produção de serapilheira e decomposição do material foliar em um cerradão na Estação Ecológica de Jataí, município de Luiz Antônio, SP, Brasil. Acta Botânica Brasileira, Belo Horizonte, v. 20, n. 1, p. 49-59, 2006.

COSTA, C. C. A. et al. Análise comparativa da produção de serapilheira em fragmentos arbóreos e arbustivos em área de caatinga na FLONA de Açu-RN. Revista Árvore, Viçosa, MG, v. 34, n. 2, p. 259-265, 2010.

DANTAS, T. V. P. et al. Florística e estrutura da vegetação arbustivo-arbórea das Areias Brancas do Parque Nacional Serra de Itabaiana/Sergipe, Brasil. Revista Brasileira de Botânica, São Paulo, v. 33, n. 4, p. 575$588,2010$.

DANTAS, T. V. P.; RIBEIRO, A. S. Estrutura populacional de Kielmeyera rugosa Choisy (Clusiaceae) no Parque Nacional Serra de Itabaiana, Estado de Sergipe. Acta Scientiarum Biological Sciences, Londrina, v. 32, p. 141-146, 2010.

EMBRAPA. Centro Nacional de Pesquisa de Solos. Sistema brasileiro de classificação de solos. 2. ed. Rio de Janeiro: EMBRAPA, 2006. 306 p.

EMBRAPA. Levantamento exploratório: reconhecimento de solos do estado de Sergipe. Recife: Embrapa; SUDENE, 1975.

FERNANDES, M. E. B.; NASCIMENTO, A. A. M.; CARVALHO, M. L. Estimativa da produção anual de serapilheira dos bosques de mangue no Furo Grande, Bragança-Pará. Revista Árvore, Viçosa, MG, v. 31, n. 5, p. 949-958, 2007.

FERREIRA, D. F. Sisvar: a computer statistical analysis system. Ciência e Agrotecnologia, Lavras, v. 35, n. 6, p. 1039-1042, 2011.

FIGUEIREDO FILHO, A. et al. Avaliação estacional da deposição de serapilheira em uma floresta ombrófila mista localizada no sul do Estado do Paraná. Ciência Florestal, Santa Maria, v. 13, 2003. p. 11-18.

HOMANN, P. S. et al. Forest soil carbon and nitrogen losses associated with wildfire and prescribed fire.

Soil Science Society of America Journal, Madison, v. 7, p. 1926-1934, 2010.

IBGE. Manual técnico da vegetação brasileira. Rio de Janeiro: IBGE, 1992. 94 p. (Série de manuais técnicos em geociências, 1).

JOBBAGY, E. G.; JACKSON, R. B. The uplift of soil nutrients by plants: biogeochemical consequences across scales. Ecology, Brooklyn, v. 85, p. 2380-2389, 2004.

KEELEY, J. E.; FOTHERINGHAM, C. J.; BAER-KEELEY, M. Determinants of post-fire recovery and succession in Mediterranean-climate shrub lands of California. Ecological Applications, Washington, v. 15, p. 1515-1534, 2005.

KNICKER, H. Vegetation fires and burnings, how does char input affect the nature and stability of soil organic nitrogen and carbon? - A review. Biogeochemistry, Malden, v. 85, p. 91-118, 2007.

KREYLING J. et al. Assisted colonization: a question of focal units and recipient localities. Restoration Ecology, Malden, v. 19, p. 433-440, 2011.

LUIZÃ O, R. C. C. et al. Variation of carbon and nitrogen cycling processes along a topographic gradient in a central Amazonian forest. Global Change Biology, Malden, v. 10, p. 592- 600, 2004.

MALAVOlTA, E.; VITTI, G. C.; OLIVEIRA, S. A. Avaliação do estado nutricional das plantas: princípios e aplicações. 2. ed. Piracicaba: POTAFOS, 1997. 319 p.

MARTINS, S. V.; RODRIGUES, R. R. Produção de serapilheira em clareiras de uma floresta estacional semidecidual no município de Campinas, SP. Revista Brasileira de Botânica, São Paulo, v. 22, p. 405-412, 1999.

MONTAGNINI, F.; JORDAN, C. F. Tropical Forest ecology: the basis for conservation and management. Berlin: Springer-Verlag, 2005.

MORELATO, L. P. C. Nutrient cycling in two southeast Brazilian forests. I. Litterfall and litter standing crop. Journal of Tropical Ecology, Winchelsea, v. 4, p. 205-215, 1993.

PROGRAMA DE MONITORAMENTO CLIMÁTICO EM TEMPO REAL DA REGIÃO NORDESTE. Precipitação. 2012. Disponível em: <http://www6.cptec.inpe.br/proclima/>. Acesso em: 10 jan. 2012.

RODRIGUES, R. R.; GANDOLFI, S. Conceitos, tendências e ações para a recuperação de florestas ciliares. In: RODRIGUES, R. R.; LEITÃO, H. F. (Ed.). Matas ciliares: conservação e recuperação. São Paulo: 
EDUSP; FAPESP, 2000. p. 235-247.

SANTANA, J. A. S. Estrutura fitossociológica, produção de serapilheira e ciclagem de nutrientes em uma área de Caatinga no Seridó do Rio Grande do Norte. 2005. 184 f. Tese (Doutorado em Agronomia) - Universidade Federal da Paraíba, Areia, 2005.

SANTANA, J.A.S.; SOUTO, J.S. Produção de serapilheira na Caatinga da região semi-árida do Rio Grande do Norte, Brasil. Idesia, Arica, v. 29, n. 2, 2011, p. 87-94.

SEDJO, R., SOHNGEN, B.; MENDELSOHN, R. Estimating carbon supply curves for global forests and other land uses. Washington: Resources for the Future, 2001. $34 \mathrm{p}$.

SOUZA, J. A.; DAVIDE, A. C. Deposição de serapilheira e nutrientes em uma mata não minerada e em plantações de bracatinga (Mimosa scabrella) e de eucalipto (Eucaliptus saligna) em áreas de mineração de bauxita. Cerne, Lavras, MG, v. 7, n. 1, p. 101-114, 2001.

SHUMACHER, M. V. Produção de serapilheira em uma floresta de Araucaria angustifolia (Bertol.) Kuntze no município de Pinhal - RS. Revista Árvore, Viçosa, MG, v. 28, n. 1, p. 29-37, 2004.

STATSOFT. Statistica data analysis system version 8.0. Tulsa: Statsoft Inc., 2008.

TERROR, V. L.; SOUSA, H. C.; KOZOVITS, A. R. Produção, decomposição e qualidade nutricional da serapilheira foliar em uma floresta paludosa de altitude. Acta Botânica Brasileira, Belo Horizonte, v. 25, n. 1, p. 113-121, 2011.

VITAL, A. R. T. et al. Produção de serapilheira e ciclagem de nutrientes de uma floresta estacional semidecidual em zona ripária. Revista Árvore, Viçosa, MG, v. 28, n. 6, p. 793-800, 2004.

VICENTE, A. et al. Levantamento Botânico. In: CARVALHO, C. M.; VILAR, J. C. (Coord.). Parque Nacional Serra de Itabaiana: levantamento da biota. São Cristóvão: UFS; Aracaju: IBAMA, 2005. p. 15-35.

WHITE, B. L. A.; RIBEIRO, A. S. Análise da precipitação e sua influência na ocorrência de incêndios florestais no Parque Nacional Serra de Itabaiana, Sergipe, Brasil. Revista Ambiente e Água, Taubaté, v. 6, p. $148-156.2011$. 